\title{
Coproporphyrin I and III Excretion in Bile and Urine
}

\author{
Neil Kaplowitz, Norman Javitt, and Attallah Kappas \\ From the Gastrointestinal Division, Department of Medicine, Cornell University \\ Medical College, and The Rockefeller University, New York 10021
}

A B S T R A C T The excretion of coproporphyrin isomers I and III was studied in the rat. Both isomers were found to bind equally to rat plasma and liver cytosol in vitro and to disappear from plasma at equal rates after single injections in vivo. During equimolar infusions of isomers into bile fistula animals, both the I and III isomers were excreted in bile in a concentration ratio of $2: 1$, respectively. Pretreatment of animals with ethinylestradiol or simultaneous infusions of phenoldibromophthalein disulfonate caused a reduction in total hepatic excretion with no change in the $2: 1$ ratio in bile. As hepatic excretion fell, excretion of both isomers in urine rose, with an increase in the proportion of the I isomer. The findings mimic those reported to occur in man and can be explained by inhibition of a common carrier which requires a stereospecific configuration that statistically favors the hepatic transport of the symmetrical coproporphyrin I isomer.

\section{INTRODUCTION}

Coproporphyrin I and III are the only two, of the four possible isomeric forms of this tetrapyrrole, which occur in nature. The coproporphyrin I isomer consists of four pyrroles condensed in a regular sequence so that the four propionic acid side groups are equidistant and the molecule is thus symmetric. In the coproporphyrin III isomer, one of the pyrroles is inverted during the biosynthesis of the porphyrin ring structure; this results in an asymmetric distribution of methyl and propionic acid side groups of the porphyrin molecule.

A discrimination between these two natural isomers appears to be made in their excretion, since they are found in unequal amounts in the urine and bile of normal man: the type III isomer predominates over the type I isomer in urine (1-6), whereas the reverse is true in bile $(7,8)$. In liver disease and in acquired or genetic disturbances characterized by reduced hepatic excretory function, there is an increase in the total uri-

Received for publication 10 May 1972 and in revised form 14 July 1972. nary excretion of coproporphyrins $(3-6,9)$, but with a concomitant shift in the isomer ratio towards a predominance of the type I compound. A series of experiments were undertaken in this study to characterize the hepatic transport process for the two coproporphyrin isomers in order to define the possible mechanism for the unequal distribution of the isomers in urine and bile during normal and impaired hepatic function.

\section{METHODS}

Male Wistar rats weighing between 250 and $350 \mathrm{~g}$ were maintained awake in restraining cages after surgical preparation with intravenous and biliary cannulae, as described previously (10). A glass funnel placed beneath the restraining cage permitted quantitative collection of urine. Ethinylestradiol $(1.25 \mathrm{mg} / 100 \mathrm{~g}$ body weight) was given in propylene glycol as described previously (11). The bile salt pool was drained overnight before constant infusions of coproporphyrin isomers and/or phenoldibromophthalein disulfonate (diBSP).

Copro I and III tetramethyl esters (Sigma Chemical Co., St. Louis, Mo., 95\% pure in our laboratory) were hydrolyzed in $8 \mathrm{~N}$ hydrochloric acid for $16 \mathrm{hr}$. After adjusting $\mathrm{pH}$ to 3.5 , the free acid was extracted into ethyl acetate. The latter was then vacuum distilled to dryness and redissolved as the infusion mixture in $0.9 \%$ sodium chloride 0.01 M phosphate buffer, $\mathrm{pH} 7.4$ (final concentration $25-100 \mu \mathrm{g} / \mathrm{ml}$ for each isomer).

Coproporphyrin isomer levels in serum, bile, and urine were determined by the thin-layer chromatographic method of Jensen (12) as modified by Moore, Stephenson, Anderson, and Schwartz (13). Extraction of bile before chromatographic separation of isomers was found to be unnecessary. Portions of 10-25 $\mu \mathrm{l}$ were placed on Silica Gel-G plates together with known standards of the coproporphyrin isomers and run in duplicate in separate tanks. After drying, the plates were viewed under UV light for location of the isomers, which were then eluted with $1.5 \mathrm{~N} \mathrm{HCl}$ and determined quantitatively in a Cary 15 spectrophotometer (Cary Instruments, Monrovia, Calif.) (absorption peak $-410 \mathrm{~nm}$ ).

Protein binding of coproporphyrin isomers was determined by equilibrium dialysis using dialyzer tubing with pore size 48 (Arthur H. Thomas Co., Philadelphia, Pa.) following previously described techniques (14). Under these conditions no binding of coproporphyrin isomers to the tubing occurred. Rat liver cytosol was prepared using the method of Levi, Gotmaitan, and Arias (15). 
TABLE I

Coproporphyrin Isomer Excretion in Rat Bile

\begin{tabular}{|c|c|c|c|c|c|c|c|}
\hline \multirow{2}{*}{\multicolumn{2}{|c|}{$\begin{array}{c}\text { Sequen- } \\
\text { tial } \\
\text { hour } \\
\text { No. }\end{array}$}} & \multicolumn{3}{|c|}{ Infusion } & \multicolumn{3}{|c|}{ Excretion } \\
\hline & & $\begin{array}{c}\text { Copro } \\
\text { I }\end{array}$ & $\begin{array}{c}\text { Copro } \\
\text { III }\end{array}$ & Ratio & $\begin{array}{c}\text { Copro } \\
\text { I }\end{array}$ & $\begin{array}{c}\text { Copro } \\
\text { III }\end{array}$ & Ratio \\
\hline & & & $\mu g / h r$ & & & $\mu g / h r$ & \\
\hline \multirow[t]{4}{*}{ A. } & 1 & 10 & 10 & 1.0 & 1.78 & 0.94 & 1.9 \\
\hline & 2 & 25 & 25 & 1.0 & 5.00 & 2.36 & 2.1 \\
\hline & 3 & 50 & 50 & 1.0 & 9.54 & 4.41 & 2.2 \\
\hline & 4 & 100 & 100 & 1.0 & 16.11 & 7.64 & 2.1 \\
\hline \multirow[t]{3}{*}{ B. } & $1 \ddagger$ & 60 & 60 & 1.0 & 9.32 & 4.62 & 2.0 \\
\hline & 1 & 30 & 60 & 0.5 & 5.41 & 5.52 & 1.0 \\
\hline & 1 & 60 & 30 & 2.0 & 7.41 & 1.75 & 4.2 \\
\hline
\end{tabular}

* Mean values for duplicate studies in 300-g animals. $\ddagger$ Each condition performed on consecutive days.

\section{RESULTS}

Equilibrium dialysis studies. Addition of $10.0 \mu \mathrm{g}$ of each isomer to $4.0 \mathrm{ml}$ rat liver cytosol, rat serum, and human serum, followed by dialysis for $16 \mathrm{hr}$ at $4^{\circ} \mathrm{C}$ against $50 \mathrm{ml} 0.01 \mathrm{M}$ phosphate buffer, $\mathrm{pH} 7.4$, indicated that both isomers were bound in the same proportions $( \pm 3 \%)$. Duplicate studies showed $42 \%$ binding to rat serum $(0.105 \mu \mathrm{g} / \mathrm{mg}$ protein $), 72 \%$ to human serum $(0.175 \mu \mathrm{g} / \mathrm{mg}$ protein $)$, and $27 \%$ to rat liver cytosol $(0.042 \mu \mathrm{g} / \mathrm{mg}$ protein $)$ for both isomers.

Excretion of coproporphyrin isomers in bile. Pairs of animals were sacrificed 10, 20, and 30 min after in- travenous injection of equimolar amounts $(64.0 \mu \mathrm{g})$ of isomers I and III. Plasma concentrations fell rapidly. Detectable amounts were found at 10 and 20 min postinjection and chromatographic analysis indicated equal concentrations of isomers I and III. By $30 \mathrm{~min}$, coproporphyrins were no longer detectable $(<0.01 \mu \mathrm{g} / \mathrm{ml})$ in plasma. Liver obtained at 30 min yielded coproporphyrin I and III in a ratio of $0.6: 1$. Bile collected from 0 to 30 min contained $72 \%$ of the injected coproporphyrin I and $35 \%$ of injected coproporphyrin III (ratio I/III = $2.1: 1$ ). Bladder urine did not contain detectable amounts of coproporphyrins.

Intravenous infusion of equimolar amounts of coproporphyrin isomers varying from 10 to $100 \mu \mathrm{g} / \mathrm{hr}$ was always followed by their excretion in bile in a $2: 1$ ratio favoring coproporphyrin I (Table I, A). Urine obtained during the study did not contain the administered isomers. During the infusion periods bile flow remained within $\pm 10 \%$ of the preinfusion rate. After the infusion periods, bile was collected for an additional $6 \mathrm{hr}$ and approximately $60 \%$ recovery of each isomer was obtained.

A change in the 2:1 excretion pattern of the isomers in bile occurred when the proportion of coproporphyrin III was increased in the infusion mixture. At a coproporphyrin I/III infusion ratio of $1: 2$, equimolar amounts of the isomers were excreted in bile (Table I, B).

Effect of ethinylestradiol on coproporphyrin cxcretion. Bile and urine collected from animals pretreated with ethinylestradiol showed changes in the amounts of isomers excreted compared to control animals (Table II).

TABLE II

Effect of Ethinylestradiol on Coproporphyrin Isomer Excretion

\begin{tabular}{|c|c|c|c|c|c|c|c|c|c|c|}
\hline \multirow{3}{*}{$\frac{\text { Condition }}{\text { Isomer }}$} & \multirow{2}{*}{\multicolumn{2}{|c|}{ Infusion }} & \multirow[b]{3}{*}{ Time } & \multirow[b]{3}{*}{ Bile flow } & \multicolumn{6}{|c|}{ Excretion } \\
\hline & & & & & \multicolumn{3}{|c|}{ Bile } & \multicolumn{3}{|c|}{ Urine } \\
\hline & I & III & & & I & III & Ratio & I & III & Ratio \\
\hline & \multicolumn{2}{|c|}{$\mu g / h r$} & & $g / h r$ & \multicolumn{6}{|c|}{$\mu g / h r$} \\
\hline Control & \multirow{2}{*}{\multicolumn{2}{|c|}{$\begin{array}{l}\text { None } \\
\text { None }\end{array}$}} & $24 \mathrm{hr}$ & 22.0 & $0.77 \ddagger$ & $0.31 \ddagger$ & 2.5 & $0.21 \ddagger$ & $0.94 \ddagger$ & 0.22 \\
\hline \multirow[t]{2}{*}{ Estrogen } & & & $24 \mathrm{hr}$ & 9.8 & $0.36 \ddagger$ & $0.12 \ddagger$ & 3.0 & $1.27 \ddagger$ & $1.56 \ddagger$ & 0.81 \\
\hline & & & $\begin{array}{c}\mathrm{Hr} \text { in } \\
\text { sequence }\end{array}$ & & & & & & & \\
\hline \multirow[t]{3}{*}{ Control } & 10 & 10 & 1 & 0.89 & 1.86 & 1.07 & 1.75 & NC\& & & \\
\hline & 25 & 25 & 2 & 0.92 & 5.19 & 2.63 & 2.0 & $\mathrm{NC}$ & & \\
\hline & 50 & 50 & 3 & 0.90 & 9.39 & 4.39 & 2.1 & $\mathrm{NC}$ & & \\
\hline \multirow[t]{3}{*}{ Estrogen } & 10 & 10 & 1 & 0.43 & 0.70 & 0.41 & 1.7 & $\mathrm{NC}$ & & \\
\hline & 25 & 25 & 2 & 0.37 & 1.46 & 0.67 & 2.2 & NC & & \\
\hline & 50 & 50 & 3 & 0.42 & 4.79 & 2.12 & 2.3 & $\mathrm{NC}$ & & \\
\hline
\end{tabular}

* Means values for pairs of animals $300 \mathrm{~g}$.

$\ddagger \mu \mathrm{g} / 24 \mathrm{hr}$.

$\S$ Not collected. 
In bile, collected over a $24 \mathrm{hr}$ period, there was a reduction in total coproporphyrin excretion with no change in the $2: 1$ ratio of the I to III isomer. In urine, however, total coproporphyrin excretion increased with a rise in the proportion of the $I$ isomer. Total urinary excretion during this study exceeded the amount that could be accounted for by reduced biliary excretion and may reflect an independent effect of ethinylestradiol on the production of coproporphyrins.

Infusion of equimolar amounts of coproporphyrin isomers showed patterns essentially similar to those obtained from the study of endogenous excretion (Table II). Thus, there was a marked reduction in the total amounts of isomers excreted in bile, but with no change in the isomer ratio.

Effect of phenoldibromophthalein disulfonate on coproporphyrin excretion. Intravenous infusion of phenoldibromophthalein disulfonate during an infusion of equimolar amounts of coproporphyrin I and III caused a marked reduction in the biliary excretion of both coproporphyrin isomers (Fig. 1). Despite a $73 \%$ reduction in total coproporphyrin excretion, the ratios of I: III in bile during three successive hourly collections were $2.2: 1$, 1.8:1, and 2.1:1 compared to a mean control ratio of 2.2: 1 before the initiation of the phenoldibromophthalein disulfonate infusion. Thus, the isomer ratio characteristic of normal bile was essentially unchanged despite the marked decrease in overall coproporphyrin excretion.

In addition, before the phenoldibromophthalein disulfonate infusion, a total of $0.21 \mu \mathrm{g}$ of coproporphyrins were excreted in urine during a $4 \mathrm{hr}$ period (ratio I: III $=0.4: 1$ ). However, during the subsequent $3 \mathrm{hr}$ infusion of the phenoldibromophthalein disulfonate, total urinary coproporphyrin excretion rose to $6.95 \mu \mathrm{g}$ with a shift in the I: III ratio from $0.4: 1$ to $1.2: 1$.

\section{DISCUSSION}

The normal pattern of coproporphyrin excretion in human bile and urine has recently been the subject of several studies $(1,2,7,8)$. It has been shown that approximately $60 \%$ of the normal daily coproporphyrin excretion is found in bile with a I to III ratio of 2.5:1. The remainder is excreted in urine with the I to III isomer ratio being approximately $0.5: 1$. With impairment of hepatic excretory function there is an increase in the total coproporphyrins excreted in urine with a rise in the proportion of the I isomer (3). This change in the pattern of coproporphyrin excretion has been found in postnecrotic cirrhosis, obstructive jaundice, hepatitis, pregnancy, and after the administration of oral contraceptive agents $(3,5,9)$. In the Dubin-Johnson syndrome $(5,6)$ a similar but greater increase in the proportion of the I isomer in urine is described (up to $90 \%$ of the total). The difference between the pattern of excretion
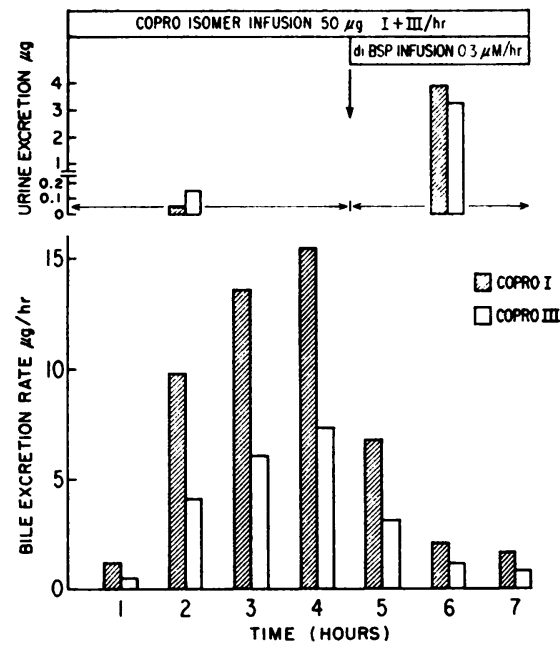

Figure 1 Effect of phenoldibromophthalein disulfonate (diBSP) on coproporphyrin (copro) isomer excretion. During an infusion of $50 \mu \mathrm{g}$ of each of the coproporphyrin isomers, the infusion of DiBSP caused a marked reduction in biliary excretion of the coproporphyrin isomers with the maintenance of a 2:1 ratio of the I to III isomers in bile. Urine coproporphyrin excretion increased during the diBSP infusion with a reversal in isomer ratio.

in this syndrome and the other states of liver impairment mentioned was found to result from an absolute reduction in total coproporphyrin III output in urine which has been attributed to a putative decrease in the activity of the enzyme, uroporphyrinogen III cosynthetase (6), in this genetic liver disease. However, this enzyme activity has not, to our knowledge, been measured in the livers of Dubin-Johnson patients. However, it is evident from the above and related findings that reduced hepatic excretion of coproporphyrin isomers may be a sensitive index of disturbed liver function which can be readily recognized by an increase in the urinary excretion of coproporphyrin I.

The reason for the differences in the proportions of the two isomers which are excreted in bile and urine is not readily apparent and was the focus for the studies reported here. A difference in protein-binding in serum favoring the ultrafilterability of the III isomer could account for a relatively greater renal excretion in normal man. However, no evidence for a difference in binding to the serum or liver cytosol fractions was found in the present study. Moreover, such a mechanism would not explain the increase in the proportion of the $I$ isomer excreted in the urine during liver disease, nor would it explain the preponderance of coproporphyrin I excreted in bile during equimolar infusions of the isomers. These findings, together with the rapid and equal rates of disappearance from plasma, suggest that a discrimination between the coproporphyrin isomers in their excretion is determined by the liver cell. 
Since neither isomer is further metabolized by the liver (16), the discrimination in hepatic excretion occurs either at the uptake or excretory step. A discrimination at the uptake step might be reflected in a change in the isomer ratio in plasma since one isomer would have a much larger volume of distribution than the other. Furthermore, selectivity at the uptake step should give rise to ratios of isomers I to III in both liver and bile that are similar. However, at a time when plasma levels of the isomers were undetectable, the ratio of coproporphyrin I to III in liver was almost the reciprocal of that in bile. Thus the proportionally greater biliary excretion of the $\mathrm{I}$ isomer is not related to a more rapid hepatic uptake process, but rather, more rapid excretion as reflected by the relative increase in the isomer remaining in liver. These findings, thus, favor the excretory rather than the uptake step as the major determinant of the unequal proportions of isomers excreted in bile.

Since the coproporphyrin isomers are organic anions, it is reasonable to consider their transport into bile within the framework of current thoughts concerning carrier transport systems which are believed to exist for a variety of other organic anions (17). Even at the relatively low infusion rates used in these studies, the concentration of each isomer in bile was greater than in plasma and their relative rates of excretion were different. The unequal excretion rates of the isomers can be attributed to either separate transport systems with different affinities for each isomer or a single carrier which apparently favors the excretion of the $I$ isomer.

The data determined from the infusion studies (Table I) do not distinguish between a single carrier and two independent carrier mechanisms for the transfer of each
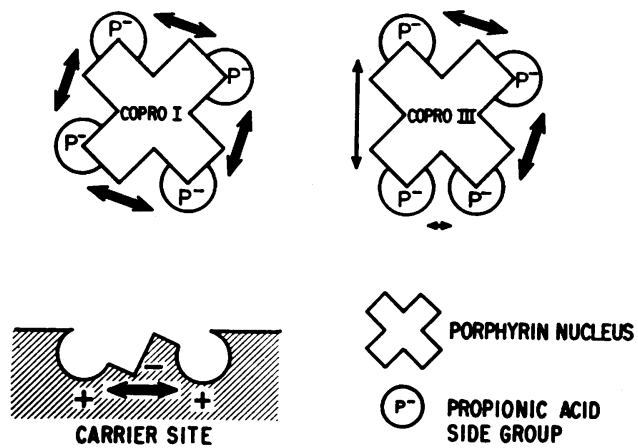

Figure 2 A model for the stereospecific hepatic transport of coproporphyrin (copro) isomers. A hypothetical transport carrier is shown (hatched area) with the thick arrow indicating the critical distance between the two points of the proposed binding site. The symmetrical isomer, coproporphyrin I, has four possibilities for binding to the carrier. Because of the reversal in position of the propionic residue (P) on one pyrrole group, the asymmetrical isomer, coproporphyrin III, has only two potential sites for binding to the carrier (corresponding to the thick arrows). stereoisomer into bile. Thus, when equimolar infusions of the isomers were administered, twice as much coproporphyrin I than coproporphyrin III was found in bile. By increasing the proportion of the III isomer in the infusion mixture, the output of coproporphyrin III is increased, with no significant decrease in isomer I excretion. Since these infusions were below the transport maximum $(\mathrm{Tm})$ for either isomer, evidence for competitive inhibition of a single carrier could not be expected.

If separate coproporphyrin carriers are present in the liver cell, however, the affinity (or efficiency of binding) of the III isomer for its transport carrier must be considerably less than that of the I isomer for its respective excretory process. Moreover, if two independent carriers were present, it is surprising that phenoldibromophthalein disulfonate, infused at rates considerably greater than that of either of the coproporphyrins, caused a proportional reduction in the excretion of each isomer in bile. Under the latter circumstances, a greater reduction in the less efficient transport system for the III isomer might have been expected; instead there was found a relatively greater reduction in the number of molecules transported per unit time in the more efficient coproporphyrin I carrier system with a resultant preservation of the usual isomer ratio in bile. Ethinylestradiol, which markedly impairs hepatic excretory mechanisms (11, $17,18)$, had a similar effect. Similarly in liver disease, alterations in the transport of the coproporphyrin I and III isomers seem inseparable; these observations, thus, all favor the view that a single carrier mechanism for both coproporphyrin isomers operates in the liver cell.

The existence of a common transport system in the liver for both the I and III isomers would readily explain the ratios found in bile and urine under normal circumstances and in the various conditions impairing hepatic excretory mechanisms. Thus, a statistical relationship between the transport of the two isomers may be envisaged in which there exists a single hepatic carrier having a two-point binding site, or fit, for the coproporphyrin molecules (Fig. 2). The two points of the binding site on the carrier may be postulated to be at a critical distance which corresponds to the distance between each of four propionic acid side groups of the symmetrical type I isomer. This isomer could, therefore, interact with the carrier in any one of four presentations corresponding to the four sides of the planar coproporphyrin I molecule. The III isomer, however, because of the reversal of the acidic substituents on one of its pyrrole rings, is asymmetrical and thus could fit the carrier in only two presentations, i.e., the two sides of the molecule having propionic acid groups at this same critical distance. Assuming equal access of the isomers to a fixed carrier site, the probability of transport of the asym- 
metric III isomer would therefore be only one-half that of the symmetrical I isomer. An impairment in the postulated common hepatic carrier transport mechanism would cause a reduction in total coproporphyrin transport into bile with consequent diversion of more of the type I than the type III compound from the biliary to the urinary route of elimination. Evidence exists that mostly coproporphyrinogen rather than coproporphyrin is excreted in urine and that oxidation then occurs (19). It is possible that the transport site in the liver cell handles the porphyrinogen as well as the porphyrin. In either event, the same statistical considerations would still apply. Thus, to explain the findings in our studies and those reported in human liver disease, it is unnecessary to postulate separate transport systems for each coproporphyrin isomer, but rather, the stereospecific requirement of a common carrier which, on a statistical basis, results in a predominance of coproporphyrin I excretion in bile.

\section{ACKNOWLEDGMENTS}

This investigation was supported by U. S. Public Health Service Grants AM 05430, AM 13094, and HD 04313.

\section{REFERENCES}

1. Aziz, M. A., S. Schwartz, and C. J. Watson. 1964. Studies of coproporphyrin. VII. Adaptation of Eriksen paper chromatographic method to the quantitative analysis of the isomers in normal human urine. J. Lab. Clin. Med. 63: 585 .

2. Koskelo, P., and I. Toivonen. 1966. Separation of urinary coproporphyrin isomers I and III by thin-layer chromatography. Studies in healthy subjects and patients with myocardial infarction. Scand. J. Clin. Lab. Invest. 18: 543 .

3. Aziz, M. A., S. Schwartz, and C. J. Watson. 1964. Studies of coproporphyrin. VIII. Reinvestigation of the isomer distribution in jaundice and liver diseases. $J$. Lab. Clin. Med. 63: 596.

4. Koskelo, P., A. Eisalo, and I. Toivonen. 1966. Urinary excretion of porphyrin precursors and coproporphyrin in healthy females on oral contraceptives. $\mathrm{Br} . \mathrm{Med}$. J. 1: 652 .

5. Koskelo, P., I. Toivonen, and H. Adlercreutz. 1967. Urinary coproporphyrin isomer distribution in the DubinJohnson syndrome. Clin. Chem. 13: 1006.
6. Ben-Ezzer, J., C. Rimington, M. Shani, U. Seligsohn, Ch. Sheba, and A. Szeinberg. 1971. Abnormal excretion of the isomers of urinary coproporphyrin by patients with Dubin-Johnson syndrome in Israel. Clin. Sci. (Oxf.). 40: 17 .

7. Aziz, M. A., and C. J. Watson. 1969. An analysis of porphyrins of normal and cirrhotic human liver and normal bile. Clin. Chim. Acta. 26: 525.

8. French, J. M., and E. Thonger. 1966. Ether-soluble porphyrins in bile, meconium and urine: a new appraisal by microscale counter-current analysis. Clin. Sci. (Oxf.). 31: 337 .

9. Koskelo, P., and I. Toivonen. 1968. Urinary excretion of coproporphyrin isomers I and III and $\delta$-aminolaevulic acid in normal pregnancy and obstetric hepatosis. Acta Obstet. Gynecol. Scand. 47: 292.

10. Javitt, N. B. and S. Emerman. 1968. Effect of sodium taurolithocholate on bile flow and bile acid excretion. $J$. Clin. Invest. 47 : 1002.

11. Harkavy, M., and N. B. Javitt. 1969. Effect of ethinyl estradiol on hepatic excretory function of the rat. In Metabolic Effects of Gonadal Hormones and Contraceptive Steroids. H. A. Salhanick, D. M. Kipnis, and R. L. Van de Wiele, editors. Plenum Publishing Corporation, New York. 11.

12. Jensen, J. 1963. Separation of the coproporphyrin isomers I and III by thin-layer chromatography. J. Chromatogr. 10: 236.

13. Moore, W. E., B. D. Stephenson, A. S. Anderson, and S. Schwartz. 1970. Detection of the heterozygous state in bovine porphyria. Analysis of urinary coproporphyrin isomers. Proc. Soc. Exp. Biol. Med. 134: 926.

14. Klotz, I. M., and F. M. Walker. 1947. The binding of organic ions by proteins. Charge and $\mathrm{pH}$ effects. $J$. Am. Chem. Soc. 69: 1609.

15. Levi, A. J., Z. Gatmaitan, and I. M. Arias. 1969. Two hepatic cytoplasmic protein fractions, $Y$ and $Z$, and their possible role in the hepatic uptake of bilirubin, sulfobromophthalein, and other anions. J. Clin. Invest. $48: 2156$

16. Rimington, C. 1965. Biliary secretion of porphyrins, and hepatogenous photosensitization. In Biliary System. W. Taylor, editor. Blackwell Scientific Publications, Ltd., Oxford. 325.

17. Schanker, L. S. 1968. Secretion of organic compounds in bile. Handb. Physiol. 5(Sect. 6) : 2433.

18. Gallagher, T. F., Jr., M. N. Mueller, and A. Kappas. 1966. Estrogen pharmacology. IV. Studies on structural basis for estrogen induced impairment of liver function. Medicine. 45: 471.

19. Rimington, C. 1963. Patterns of porphyrin excretion and their interpretation. S. Afr. J. Lab. Clin. Med. 9: 255. 